\title{
Microdosing Psychedelics: Personality, mental health, and creativity differences in
}

\section{microdosers}

Thomas Anderson, Rotem Petranker, Daniel Rosenbaum, Cory R. Weissman, Le-Anh DinhWilliams, Katrina Hui, Emma Hapke, \& Norman A. S. Farb

Accepted to Psychopharmacology, December 2018

Published as: Anderson, T., Petranker, R., Rosenbaum, D., Weissman, C. R., Dinh-Williams, L.-A., Hui, Hapke, E., \& Farb, N. A. S. (2019). Microdosing psychedelics: personality, mental health, and creativity differences in microdosers. Psychopharmacology.

https://doi.org/10.1007/s00213-018-5106-2

\begin{abstract}
Microdosing psychedelics — the regular consumption of small amounts of psychedelic substances such as LSD or psilocybin — is a growing trend in popular culture. Recent studies on full-dose psychedelic psychotherapy reveal promising benefits for mental well-being, especially for depression and end-of-life anxiety. While full-dose therapies include perception-distorting properties, microdosing may provide complementary clinical benefits using lower-risk, nonhallucinogenic doses. No experimental study has evaluated psychedelic microdosing, however; this pre-registered study is the first to investigate microdosing psychedelics and mental health. Recruited from online forums, current and former microdosers scored lower on measures of dysfunctional attitudes and negative emotionality and higher on wisdom, open-mindedness, and creativity when compared to non-microdosing controls. These findings provide promising initial evidence that warrants controlled experimental research to directly test safety and clinical efficacy. As microdoses are easier to administer than full-doses, this new paradigm has the exciting potential to shape future psychedelic research.
\end{abstract}




\section{Introduction}

Microdosing psychedelics — the practice of regularly consuming very low doses of psychedelic substances such as lysergic acid diethylamide (LSD) or psilocybin ("magic" mushrooms) - is a growing practice despite a lack of scientific research validating its effects. One online microdosing forum (/r/microdosing subreddit, Reddit Inc, San Francisco, CA, USA) has approximately 30,000 subscribers and doubled its subscriber count in the past year (Figure 1). The popular media has described consumption of psychedelics in doses much lower than typical therapeutic doses (Fadiman, 2011; Leonard, 2015; Solon, 2016; Waldman, 2017) and articles and anecdotes claim benefits including improved mood, focus, and creativity alongside decreased symptoms of depression and anxiety. While decreased depression and anxiety are consistent with research on full-dose psychedelics (Carhart-Harris et al., 2017; Griffiths et al., 2016), microdosing could offer these benefits without any perceptual distortions and reduced need for expensive clinical oversight typical of full-dose psychedelic psychotherapy. Nevertheless, both LSD and psilocybin are controlled substances in most countries and so members of the public enticed by purported benefits of microdosing expose themselves to the risks implied by criminalized activity. Such risks are exacerbated by an absence of even minimal scientific evidence that normally surrounds clinical use, such as data on safety, efficacy, common side-effects, contraindications, and appropriate dose and dose schedule.

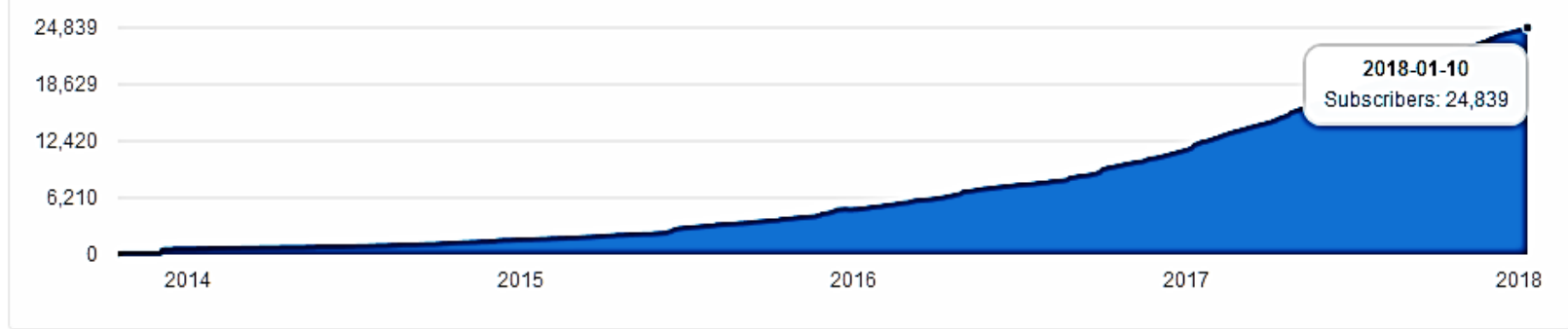

Figure 1. Rise in subscribers to an online microdosing forum, Reddit. 
It is unlikely that normative standards for microdosing will emerge without an initial description of current microdosing practices and associated outcomes. We therefore measured self-reported practices and psychological function of participants in existing microdosing communities and compared them to control participants with no microdosing experience. This design allows for a structured description of the common practices used in microdosing from which future clinical trials can build.

\subsection{Full-dose Psychedelics}

Interest in microdosing is likely predicated on research linking clinical benefits to fulldose psychedelic use. By 1975, over one thousand studies had linked psychedelic substance use with salutary effects on mental health and personal growth (Greenspoon \& Bakalar, 1979). More recent research suggests efficacy for a number of health conditions, including obsessive compulsive disorder (Moreno et al., 2006), alcohol dependence (Bogenschutz et al., 2015), tobacco dependence (Johnson et al., 2014), depression (Carhart-Harris et al., 2017; Osório et al., 2015), and end-of-life anxiety (Griffiths et al., 2016; Ross et al., 2016).

While research on psychedelics provides evidence for the therapeutic effects of fulldoses, such experiences are often quite intense thus confer substantive participant risk. Popular vernacular includes the term "bad trip", and, indeed, one study participant described a full-dose experience as "the worst experience of her life" (Griffiths et al., 2011). Although research on LSD and psilocybin suggest low risks for abuse or harmful effects (Amsterdam et al., 2011; Halpern and Pope, 1999), a small percentage of users are at risk of developing persisting perceptual effects (Hallucinogen Persisting Perception Disorder; Martinotti et al., 2018) or risk being hospitalized for acute intoxication, especially if mixed with alcohol (Hardaway et al., 2016). While large population studies suggest that psychedelics are not usually associated with 
detrimental mental health outcomes (Krebs \& Johansen, 2013), microdosing may circumvent this issue as anecdotal reports suggest numerous positive outcomes without the risks associated with acute full-dose intoxication (Fadiman, 2011).

\subsection{The Present Study}

In this study we describe the psychological profile of the growing microdosing community by making comparisons against a population of non-microdosers. We compared groups of self-described microdosers (current and former microdosers) against controls (no microdosing experience) across a variety of mental health and personality variables. These include dysfunctional attitudes (de Graaf et al., 2009), wisdom (Glück et al., 2013), negative emotionality and open-mindedness (Soto and John, 2016), and creativity (Silvia, 2011). This study is part of a larger project that also reports on the demographics and psychiatric history of microdosing users (Rosenbaum et al., 2018). A qualitative report examining subjective benefits and drawbacks of microdosing is also in preparation (Anderson et al., 2018). We presently address pre-registered hypotheses about the outcomes associated with microdosing experience on validated scales.

\section{Methods}

\subsection{Pre-Registered Hypotheses}

Prior to data collection this study was pre-registered on the Open Science Framework

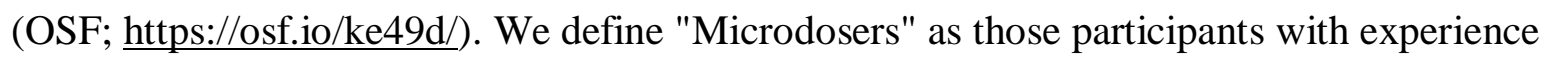
microdosing, whether current or former use. We pre-registered the following hypotheses:

\section{Mental Health Vulnerability, Wisdom, and Personality}

While the mechanisms driving psychedelic substances' clinical efficacy are unclear, several psychological constructs are likely involved. These include practical indicators of 
flourishing, such as freedom from dysfunctional beliefs about oneself, other people, and the world; wisdom; and personality traits, especially neuroticism and openness to experiences.

Psychedelic substances are purported to have profound effects on one's understanding of the self and world, leading to enhanced insight and personal growth (Domínguez-Clavé et al., 2016; Dos Santos et al., 2016; Kometer et al., 2015; Strassman, 2016). As such, we hypothesized that microdosers would have lower dysfunctional attitudes and higher wisdom than nonmicrodosers. Furthermore, improved mood and reduced mental health concerns are commonly reported outcomes of microdosing online (/r/microdosing subreddit, Reddit Inc, San Francisco, CA, USA). For this reason, we hypothesized that microdosers would have lower negative emotionality (depression, anxiety, and emotional volatility) than non-microdosers. Finally, participants experiencing a single full dose of psilocybin showed a robust and sustained increase in openness (MacLean et al., 2011); we therefore predicted that microdosers would also have higher openness.

H1a: Microdosers will have lower dysfunctional attitude scores than non-microdosers. H1b: Microdosers will have higher wisdom scores than non-microdosers.

H1c: Microdosers will have lower negative emotionality scores than non-microdosers. H1d: Microdosers will have higher open-mindedness scores than non-microdosers.

\section{Creativity}

The Broaden and Build theory (Fredrickson, 2004) proposes a link between positive emotions and relaxed cognitive constraints as improved well-being may recruit personal resources in the generation of creative ways of coping with challenges. Enhanced creativity is one of the commonly reported outcomes of microdosing in media reports (Solon, 2016) and 
online (/r/microdosing subreddit, Reddit Inc, San Francisco, CA, USA) and is often reported as a benefit of full-dose psychedelics (Fadiman, 2011).

H2: Microdosers will have higher creativity scores than non-microdosers.

\section{Importance of Benefits}

Participants were asked to rate how important qualitative benefits of microdosing were to them. We used this measure of subjective "importance of benefits" as a broad outcome of participants' positive valuation of microdosing. Based on online anecdotal reports we predicted that there would be a total-dose response curve such that microdosers would rate the importance of benefits as quickly increasing to a plateau. Concerning dose scheduling, James Fadiman (2011) proposed a dose-schedule such that microdosers consume their substance one day, then refrain for two days, then dose again; we hypothesized that this schedule would show optimal reported importance of benefits compared to alternate dose frequencies, perhaps due to substance-tolerance (more frequent) or limited efficacy (less frequent).

\section{Total Doses and Dose Frequency}

H3a: A logarithmic relationship will exist between total lifetime microdoses and average reported importance of benefits. Specifically, benefits are expected to be minimal with minimal total doses, then increase, and subsequently stabilize at a plateau.

H3b: A quadratic relationship will exist between frequency of microdosing and average reported importance of benefits. Specifically, maximum benefits are expected when participants report frequency of microdoses at $\sim 3$ days between microdoses with reduced benefits for shorter and longer frequencies.

More frequent and more intense positive experiences with a substance motivate future use of that substance (de Wit and Phillips, 2012). As such, microdosers with a more extensive 
history of full-dose psychedelic use may be especially motivated to try microdosing and may evaluate benefits of microdosing more highly. This positivity bias may extend beyond psychedelics as, more generally, substance use is associated with greater openness to experience (Gunnarsson et al., 2008; Terracciano et al., 2008; Trull and Sher, 1994), which may include an openness to try novel pharmacological interventions, such as microdosing.

\section{Substance-Use History}

H4a: Microdosers reporting at least one life-time use of a classic psychedelic (LSD, psilocybin mushrooms, DMT, ayahuasca, mescaline) at full dose will report higher average importance of benefits than microdosers that have not had a full dose.

H4b: Microdosers reporting greater variety of recreational substance use ("Polydrug user experience index", see below) will report higher average importance of benefits than microdosers with less recreational substance experience.

\subsection{Deviations from Pre-Registration}

A survey-flow error resulted in unintended data-collection on dose frequency and importance ( $\mathrm{H} 3$ and $\mathrm{H} 4)$ from participants with no experience microdosing; this data has been discarded.

\subsection{Participants}

Participant were snowball-recruited via social media (e.g. Facebook, Twitter) and recruited through posts on the online forum "reddit" (Reddit Inc, San Francisco, CA, USA): links were posted under the username /u/oredna on the following subreddits: Microdosing, Nootropics, Psychonaut, RationalPsychonaut, Tryptonaut, Drugs, LSD, shrooms, DMT, researchchemicals, and SampleSize. Both participants with experience and participants without experience 
microdosing psychedelics were recruited for this study. Participation was voluntary, and participants were not remunerated. The survey was in English and internationally available.

Participants exited the online survey at different stages of completion; different analyses therefore employ different numbers of participants. While 1390 respondents began the survey, many 475 exited before responding, 3 requested that their responses be removed, and 3 responses were removed for disingenuous responding, i.e. "trolling". In total 909 participants entered enough data to be included in analyses, sorted into two categories: those with microdosing experience (Microdosers: $n=594,65 \%$ ) and those without such experience (Non-microdosers: $n$ $=315,35 \%$ ). Of these participants $29 \%$ were currently microdosing (current microdosers), $37 \%$ had microdosed in the past but have since stopped (former microdosers), $30 \%$ were interested in microdosing but had no prior experience, and $4 \%$ had no prior experience and reported not being interested in microdosing. Participants from 29 countries responded to the survey (median age $=$ $26,82 \%$ males, $70 \%$ white). For a more comprehensive breakdown see the full epidemiological report (Rosenbaum et al., 2018).

\subsection{Design and Questionnaires}

Following informed consent, participants completed online computer-based questionnaires (https://osf.io/jmcrh/) including questions pertaining to microdosing habits (substance, frequency, dosage), substance use and mental health history, dispositional personality variables (Dysfunctional Attitudes, Wisdom, Negative Emotionality, Open-Mindedness), and a creativity task. Questions were displayed according to experience with microdosing, i.e. individuals who reported never having microdosed were not shown questions related to a history of microdosing (note survey flow error, 1.4.1 above). For uniformity, all scales were rated using a continuous 0-100 slider-scale with nominal descriptors at 0 ("Disagree Strongly") and 100 
("Agree Strongly") (Matejka et al., 2016). For brevity, methods reported here focus on variables analyzed in this paper; a complete list of all questions is available on the OSF pre-registration.

\subsubsection{Microdosing Substance}

The majority of participants reported using LSD (65\%) and/or Psilocybin (28\%) for microdosing; $16 \%$ reported using another substance. For a more comprehensive breakdown see the full epidemiological report (Rosenbaum et al., 2018).

\subsubsection{Mental Health Vulnerability}

The DAS-A-17 is a short-version of the Dysfunctional Attitude Scale, a 40-item selfreport scale designed to measure the presence and intensity of dysfunctional beliefs (de Graaf et al., 2009). Participants rate statements of beliefs (e.g. "If I fail at my work, then I am a failure as a person.") on a 7-point Likert scale and the total score is the sum of the 17-items (range: 17119) with higher scores indicating more dysfunctional attitudes (Weissman and Beck, 1978). The DAS-A-17 includes a total-score and two subscales: "perfectionism/performance evaluation" (11 items) and "dependency" (6 items). Reliability for total score was excellent $(\alpha=0.91)$ and good for the subscales (perf: $\alpha=0.87$, dep: $\alpha=0.85$ ).

A Mental Health Index of psychological disorders was computed as a simple binary 0/1 based on the question, "Have you ever been diagnosed by a doctor or health care professional (e.g., psychiatrist, psychologist) with any of the following diagnoses", which was followed by a list of DSM-V diagnoses. Endorsing any diagnosis was coded as a "1", otherwise "None of the above" was coded as "0". Comprehensive findings will be available in the epidemiological report (Rosenbaum et al., 2018). 


\subsubsection{Wisdom}

The Brief Wisdom Screening Scale (BWSS, (Glück et al., 2013) was developed by selecting the 20 items that were most highly correlated with the common factor of "wisdom selfreport" across three leading wisdom self-report measures. Reliability was good $(\alpha=0.86)$.

\subsubsection{Personality}

The Big Five Inventory 2 (BFI2, Soto and John, 2016) is an updated five-factor personality measure using the commonly recognized five-factor model: Extraversion, Agreeableness, Conscientiousness, Negative Emotionality (formerly “Neuroticism”), and OpenMindedness (formerly Openness to Experience). Our hypotheses were centered on two of these subscales: Negative Emotionality and Open-Mindedness, thus only these factors were measured. Reliability was good (Negative Emotionality $\alpha=0.91$, Open-Mindedness $\alpha=0.79$ ).

\subsubsection{Creativity}

The Unusual Uses Task is a task in which participants generate creative uses for mundane objects (UUT; (Silvia, 2011). The UUT instructions emphasized the importance of original responses, reading "Please try and think of the most unusual, creative, and uncommon uses you can imagine" (Harrington, 1975). Participants were asked to give as many responses as they could for each of two items (brick, knife), allotted one minute for each. During analysis, responses were split into alphabetical lists to avoid within-participant biases. Responses were rated by three independent research assistants using three dimensions: uncommon, clever, and remote (Silvia et al., 2008). Dimension-scores across objects were averages to produce three dimension-scores. Intraclass correlation coefficient was moderate for each dimension (Uncommon: 68.25, Remote: 57.25, Clever: 59.75) and moderate for each object (brick $=.61$, knife $=.64)$. 


\subsubsection{Importance of Benefits}

Participants rated qualitative benefits of microdosing in terms of personal, subjective importance for three self-generated benefits. The average of these scores was then used as a broad index of participants' subjective valuation of microdosing, analyzed in H3/4 in this study. A taxonomy that organizes the participant-generated benefits has been generated using Grounded Theory analysis and will be featured in an independent report (Anderson et al., 2018).

\subsubsection{Microdosing Frequency}

Microdosers reported the total number of lifetime microdoses taken ( 0 to 100$)$. They also reported dose scheduling, that is, the number of days spaced between each microdose (dose every day to dose once every two months).

\subsubsection{Substance-Use History}

To test $\mathrm{H} 4$ concerning substance-use history, participants reported their experience with full-dose psychedelics and with other substances. We developed a novel index: the "Polydrug User Experience Index". This novel measure was computed as the sum of recreational experiences across 13 classes of substance (e.g. Alcohol, Cannabis, MDMA, Stimulants, Opiates, Dissociatives, etc.) accounting for recency of experience. Each class of substance was scored according to the following metric: (a) used in past month: +4 points; (b) used in past year: +2 points; (c) used ever: +1 point; (d) never used: +0 points; (e) Prefer not to answer: +0 points. Scores range from $0-52$, with lower scores indicating less experience with recreational substance use. 


\subsubsection{Mood}

A "Valence" score was computed using a mood-board (https://osf.io/jmcrh/) as the count of pleasant minus unpleasant items, as was an "Arousal" score for high-intensity minus lowintensity moods.

\section{Results}

Table 1. Contrast between Microdosers and Non-Microdosers, means with standard deviations and standardized effect-sizes.

\begin{tabular}{|c|c|c|c|}
\hline Variable & Microdoser & Non-Microdoser & $\begin{array}{l}\text { Effect size } \\
\text { d }[95 \% \mathrm{CI}]\end{array}$ \\
\hline Age & $27.23(8.94)$ & $26.36(7.78)$ & $0.1[-0.04,0.25]$ \\
\hline Education $^{1}$ & $4.72(1.72)$ & $4.78(1.77)$ & $-0.03[-0.18,0.11]$ \\
\hline $\mathrm{SES}^{2}$ & $0.50(1.33)$ & $0.51(1.40)$ & $-0.01[-0.15,0.14]$ \\
\hline Mood - Valence & $2.33(4.40)$ & $-0.16(4.14)$ & $0.58[0.44,0.72]$ \\
\hline Mood — Intensity & $-0.08(2.42)$ & $0.02(2.40)$ & $-0.04[-0.18,0.09]$ \\
\hline Dysfunctional Attitudes (DAS-17) & $40.62(16.28)$ & $49.30(16.33)$ & $-0.53[-0.77,-0.29]$ \\
\hline Wisdom (BWSS) & $66.68(13.16)$ & $60.05(12.98)$ & $0.51[0.27,0.74]$ \\
\hline Negative Emotionality (BFI-2) & $41.53(20.06)$ & $48.16(18.89)$ & $-0.34[-0.56,-0.11]$ \\
\hline Open-Mindedness (BFI-2) & $76.43(12.44)$ & $73.33(13.16)$ & $0.25[0.02,0.47]$ \\
\hline
\end{tabular}


1 - Education was coded according to the International Standard Classification of Education (UNESCO Institute for Statistics, 2011): ISCED level 0 = Early childhood education, $1=$ Primary education, $2=$ Lower secondary education, 3 = Upper secondary education, 4 = Post-secondary non-tertiary education Tertiary education, $5=$ Short-cycle tertiary education, $6=$ Bachelor's or equivalent level, $7=$ Master's or equivalent level, $8=$ Doctoral or equivalent level

2 - Socio-Economic Status (SES) was coded as: -3 = Non-working class (casual workers, pensioners, or dependents); -2 = Working class (semi-skilled or unskilled manual workers); -1 = Skilled working class (skilled manual workers); 0 = Lower-middle class (junior managerial, administrative, or professional); 1 $=$ Middle class (intermediate managerial, administrative, or professional); $2=$ Upper-middle class (higher managerial, administrative, or professional); $3=$ Upper class (royalty or immense heritable wealth).

$3-D A S$ scores have been transformed to the original DAS-17 scale (17-119).

Table 2. Follow-up Analysis of Microdosers (Current versus Former), means with standard deviations and standardized effect-sizes.

\begin{tabular}{|l|c|c|c|}
\hline Variable & $\begin{array}{c}\text { Current } \\
\text { Microdoser }\end{array}$ & $\begin{array}{c}\text { Former } \\
\text { Microdoser }\end{array}$ & $\begin{array}{c}\text { Effect size } \\
\text { d [95\% CI] }\end{array}$ \\
\hline Age & $28.89(9.71)$ & $25.93(8.06)$ & $\mathbf{0 . 3 3}[\mathbf{0 . 1 6}, \mathbf{0 . 5 1}]$ \\
\hline Education & $4.83(1.70)$ & $4.64(1.73)$ & $0.11[-0.06,0.28]$ \\
\hline SES & $0.48(1.34)$ & $0.52(1.32)$ & $-0.03[-0.2,0.14]$ \\
\hline Mood — Valence & $2.93(4.57)$ & $1.86(4.21)$ & $\mathbf{0 . 2 4}[\mathbf{0 . 0 8}, \mathbf{0 . 4 1}]$ \\
\hline Mood - Intensity & $0.10(2.38)$ & $-0.23(2.45)$ & $0.14[-0.03,0.3]$ \\
\hline Dysfunctional Attitudes (DAS-17) & $39.53(14.58)$ & $41.53(17.56)$ & $-0.12[-0.36,0.11]$ \\
\hline Wisdom (BWSS) & $66.09(12.86)$ & $67.18(13.42)$ & $-0.08[-0.32,0.15]$ \\
\hline
\end{tabular}




\begin{tabular}{|l|c|c|c|}
\hline Negative Emotionality (BFI-2) & $43.32(19.98)$ & $39.95(20.07)$ & $0.17[-0.06,0.4]$ \\
\hline Open-Mindedness (BFI-2) & $77.06(11.91)$ & $75.87(12.91)$ & $0.09[-0.13,0.32]$ \\
\hline
\end{tabular}

\subsection{Pre-Registered Hypotheses and Planned Follow-up Analysis}

\subsubsection{Mental Health Vulnerability, Wisdom, and Personality}

Mental Health Vulnerability. Microdosing predicted lower scores on Dysfunctional Attitudes $(\mathrm{b}=-8.69,95 \%$ CI [-12.48 -4.89], $\mathrm{z}(364)=-4.49, \mathrm{p}<.001, \mathrm{r}=-0.92)$, even when controlling for a history of mental illness, which was also significant $(b=5.74,95 \%$ CI [2.45

9.03], $\mathrm{z}(364)=3.42, \mathrm{p}<.001, \mathrm{r}=0.85)($ Figure 2). Dysfunctional Attitudes were not related to current versus former microdosing $(b=1.90,95 \%$ CI [-1.91 5.71], $\mathrm{p}=0.33)$, nor to type of substance used (LSD vs Psilocybin: $\mathrm{b}=0.56,95 \%$ CI [-4.93 6.05], $\mathrm{p}=0.842$ ), nor to total number of lifetime microdoses $(b=-1.66,95 \%$ CI [-3.47 0.15], $\mathrm{p}=0.074)$. 


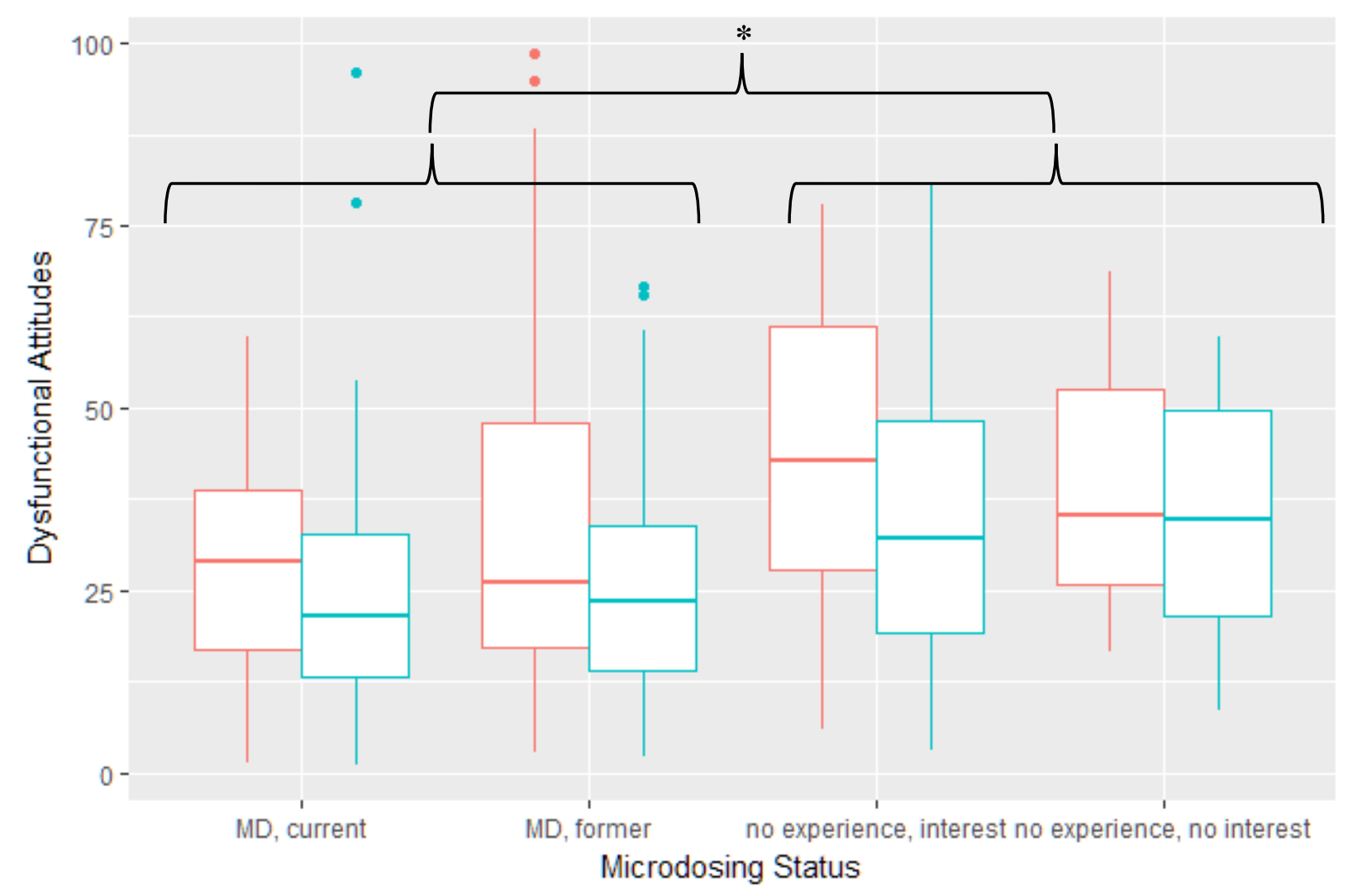

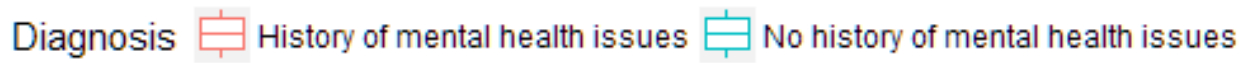

Figure 2. Differences in dysfunctional attitudes between microdosing status

Wisdom. Microdosing predicted higher wisdom scores $(b=6.61,95 \%$ CI [3.52 9.69], $\mathrm{z}(367)=4.19, \mathrm{p}<.001, \mathrm{r}=0.88)$ when controlling for age and level of education, which were not significant (age: $\mathrm{b}=-0.11,95 \%$ CI [-0.26 0.04], $\mathrm{p}=0.16$, education: $\mathrm{b}=0.40,95 \%$ CI [-0.43 1.24], $\mathrm{p}=0.35$ ). No significant differences were found between current and former microdosers $(\mathrm{b}=1.09,95 \% \mathrm{CI}[-1.964 .13], \mathrm{p}=0.48)$, nor type of substance used $(\mathrm{b}=1.37,95 \%$ CI [-2.83 5.57], $\mathrm{p}=.523)$, nor total lifetime number of microdoses $(\mathrm{b}=0.62,95 \% \mathrm{CI}[-0.802 .05], \mathrm{p}=$ $0.39)$.

Negative Emotionality. Microdosing predicted lower Negative Emotionality $(b=-5.78$, $95 \% \mathrm{CI}[-10.13-1.43], \mathrm{z}(396)=-2.60, \mathrm{p}=.009, \mathrm{r}=-0.85)$, even after controlling for gender, 
which was also a significant predictor (higher Negative Emotionality in females, $b=10.49,95 \%$ CI $[5.33$ 15.65], $\mathrm{z}(396)=3.99, \mathrm{p}<.001, \mathrm{r}=0.95)$. Planned follow-up analysis tested the difference between current and former microdosers and no significant difference existed between the groups $(b=-2.95,95 \%$ CI [-7.47 1.58], $p=0.20)$, nor between substance used $(b=-5.18$, $95 \%$ CI [-11.50 1.15], $\mathrm{p}=.110)$, nor any effect of lifetime number of microdoses $(b=-0.25,95 \%$ CI [-2.38 1.89], $\mathrm{p}=0.82$ ) on Negative Emotionality.

Open-Mindedness. Microdosing predicted greater Open-Mindedness $(b=3.24,95 \%$ CI $[0.386 .10], \mathrm{z}(392)=2.22, \mathrm{p}=.027, \mathrm{r}=0.67)$, including when controlling for education, which was not significant $(b=0.08,95 \%$ CI $[-0.650 .81], p=0.83)$. Again there were no significant differences between current and former microdosers $(b=-1.18,95 \%$ CI [-4.00 1.64], $p=0.41)$, nor type of substance used $(b=1.35,95 \%$ CI [-2.61 5.31], $\mathrm{p}=.506)$, nor total lifetime number of microdoses $(b=0.77,95 \%$ CI [-0.55 2.09], $p=0.26)$.

\subsubsection{Creativity}

Microdosing predicted higher scores on all three creativity facets: on average, responses made by microdosers were more clever $(b=0.57, \mathrm{SE}=0.13, \mathrm{z}(423)=4.25, \mathrm{p}<.001, \mathrm{r}=0.15)$, more uncommon $(\mathrm{b}=0.50, \mathrm{SE}=0.15, \mathrm{z}(427)=3.42, \mathrm{p}<.001, \mathrm{r}=0.14)$ and more remote $(\mathrm{b}=$ $0.74, \mathrm{SE}=0.16, \mathrm{z}(425)=4.49, \mathrm{p}<.001, \mathrm{r}=0.20)$

\subsubsection{Importance of Benefits}

Self-reported "importance of benefits" was intended to reflect participants' broad valuation of microdosing. Counter to $\mathrm{H} 3 \mathrm{a} / \mathrm{b}$, no significant differences were found in reported importance of benefits when regressed on lifetime microdoses $(b=1.01,95 \%$ CI [-0.81 2.82], $p$ $=.277$ ) nor frequency of microdosing regardless of explored linear and non-linear relationships (raw: $\mathrm{b}=-0.07,95 \%$ CI [-0.66 0.53], $\mathrm{p}=0.83$; squared: $\mathrm{b}=-0.0004,95 \%$ CI [-0.01 0.01], $\mathrm{p}=$ 
0.94; logarithmic: $\mathrm{b}=1.21,95 \%$ CI [-2.68 5.09], $\mathrm{p}=0.54)$. Counter to $\mathrm{H} 4 \mathrm{a} / \mathrm{b}$ there were also no significant difference in the importance of benefits between participants who had previous experience with full-dose classic psychedelics and those who had no such experience $(b=-4.09$, $95 \% \mathrm{CI}[-11.803 .61], \mathrm{p}=0.30)$ nor based on the variety and recency of recreational substance use (Polydrug User Experience Index: $b=-0.006,95 \%$ CI [-0.26 0.25], p = 0.96).

\subsection{Exploratory Analysis}

Exploratory comparison of mood measures (valence and intensity) by Welch's t-test revealed that microdosers reported significantly more positive valence $(\mathrm{M}=2.33, \mathrm{SD}=4.40)$ than non-microdosers $(\mathrm{M}=-0.16, \mathrm{SD}=4.14$; difference: $2.49,95 \% \mathrm{CI}[1.913 .07], \mathrm{t}(675)=8.44, \mathrm{p}<$ $.001, \mathrm{r}=0.31$ ). No differences were found for mood intensity (difference: $-0.11,95 \% \mathrm{CI}[-0.44$ $0.22] \mathrm{p}=0.53)$. For valence, current microdosers $(\mathrm{M}=2.93, \mathrm{SD}=4.57)$ also reported more positive valence than former microdosers $(\mathrm{M}=1.86, \mathrm{SD}=4.21$; difference: $1.07,95 \% \mathrm{CI}[0.35$ 1.79], $\mathrm{t}(533)=2.92, \mathrm{p}=.004, \mathrm{r}=0.13)$, but no difference in mood intensity (difference: 0.33 , $95 \%$ CI [-0.06 0.72], $\mathrm{p}=0.10)$.

\section{Discussion}

This is the first preregistered report on microdosing psychedelics and is intended to inform future lab-based clinical intervention studies. We investigated psychedelic microdosing in online communities and tested pre-registered hypotheses (https://osf.io/ke49d/) concerning the relationship between experience with microdosing and various mental health and personality variables. Our results suggest a beneficial relationship wherein experience with microdosing is associated with lower dysfunctional attitudes and negative emotionality and higher wisdom, open-mindedness, and creativity. The most popular substances used to microdose were LSD and psilocybin, and no significant differences were found based on substance choice. Hypotheses 
predicting perceived importance of microdosing from dose-related practices were unsupported and optimal dose scheduling remains an open question. Exploratory analyses revealed that microdosers, especially current microdosers, had more positive emotional valence than nonmicrodosers, whereas emotional intensity was not significantly different. Taken together, these findings suggest that randomized, placebo-controlled clinical trials (RCTs) of microdosing are warranted to investigate the causal efficacy of microdosing.

Consistent with our hypotheses, microdosing experience was associated with meaningfully lower levels of dysfunctional attitudes. Individuals with higher dysfunctional attitudes maintain a set of disadvantageous beliefs that increase vulnerability to stressors (Jarrett et al., 2012) and high scores are associated with depression (Adler et al., 2015; de Graaf et al., 2009). Also consistent with our hypotheses was the lower negative emotionality seen in microdosers, though the estimated effect was less precise. Tendencies to experience negative emotionality (e.g. anxiety, depression, emotional volatility) are a robust predictor of mental and physical health problems (Lahey, 2009) thus reduced vulnerability is reflected in the lower scores seen in microdosers. Exploratory analysis revealed that microdosers had more positive emotional valence than non-microdosers, linking microdosing to better mood states. While causation cannot be inferred from these results, significant differences were preserved even after controlling for potent covariates, such as gender and history of mental illness, indicating a potentially distinct contribution of microdosing on mental health vulnerability that warrants further study.

Microdosers also had higher wisdom, which is a complex trait (BWSS, Glück et al., 2013). As measured by the BWSS, wisdom is understood to reflect learning from one's mistakes, considering multiple perspectives when facing a situation, being in tune with one's own emotions 
and the emotions of others, and feeling a sense of connection and unity. Higher scores, as seen in this sample of microdosers, may be associated with cognitive and emotional processing differences including enhanced capacity for perspective taking, resilience in the face of the vicissitudes of life, and increased feelings of engagement and connection. RCT research addressing the relationship between wisdom and microdosing are warranted.

Greater open-mindedness was expected in microdosers compared to controls due to previous studies noting increases in openness following a full-dose of psilocybin (MacLean, Johnson, \& Griffiths, 2011). These differences were supported, though this effect was relatively weaker than the others. Still, given the findings from full-dose psychedelic studies, future clinical intervention research should continue investigating any causal relationship between openmindedness and microdosing.

Microdosers were more creative when finding unusual uses for household items. This is consistent with Fredrickson's (2004) Broaden and Build theory, which suggests a positive relationship between creativity and positive affect, which was also seen in microdosers. Happier, more creative people may be more likely to apply novel modes of thinking in their personal and interpersonal challenges (Fredrickson, 2004). Our findings are also consistent with the anecdotal reports that a relationship between microdosing, creativity, and mood exists, but RCTs, ideally with multiple creativity measures, are required.

None of our hypotheses concerning the importance of microdosing benefits and microdosing practices were supported. It is likely that this measure was not sensitive and specific enough; planned analyses of qualitative benefits and drawbacks of microdosing will be explored in a separate report (Anderson et al., 2018). It may be that microdosing frequency is truly unrelated to the subjective valuation of microdosing, but this seems improbable. We suggest that 
this research question is best addressed in RCT studies focused on specific benefits with experimental manipulation of dose and schedule to determine optimal benefit-specific protocols. Similarly, there is no evidence that outcomes are predicated on prior experience with substances, whether full-dose psychedelics or with a variety of substances. More microdosers had experience with full-doses $(69 \%, \mathrm{n}=412)$ then $\operatorname{did} \operatorname{not}(31 \%, \mathrm{n}=182)$ and many microdosers (and nonmicrodosers) had experience with full-dose psychedelics within the month prior to completing the survey. As full-dose psychedelics can have benefits lasting at least a month (Carhart-Harris et al., 2017) this covariate should be formally modelled in future microdosing research designs, which should aim to include both psychedelic-naïve and psychedelic-experienced participants.

\section{Limitations and Future Directions}

The sample is both a strength and a limitation of this study. This sample represents a true community of microdosers with dozens of countries represented, however, countries in the Anglo cultural cluster make up the majority of the sample (>70\%) and participants were predominantly middle-class, white, male, and heterosexual. Sampling from online communities, including Reddit, could create a demographic bias thus we cannot suggest a definitive epidemiological generalization. Despite this limitation, this sample does inform us about real community practices in an otherwise unstudied population and reflects our sample of interest.

A second limitation of this study is its correlational nature. This cross-sectional design contained no longitudinal component or experimental manipulation and cannot be used to infer causal relationships. Our findings of group-differences do not infer that microdosing caused these differences as some of the measured constructs may even promote an increased willingness to explore microdosing, e.g. open-mindedness. Instead, these findings are intended as a 
descriptive foundation upon which experimental and clinical studies of psychedelic microdosing can be designed, exploring the directionality of relationships established in the present study.

To test causal hypotheses concerning microdosing effects, pre-registered randomized placebo-control trials (RCTs) are needed. With random assignment to microdose or placebo it would be possible to determine whether microdosing causally influences mental health and personality. Following positive causal findings, mechanistic studies could then investigate the observed efficacy in terms of physiological, psychological, and neurobiological changes.

Promisingly, microdosing may prove easier to administer, monitor, and placebo-control in lab settings due to the absence of the intense perceptual shifts induced by full-doses. Microdosing may thus be amenable to designs that could aid in mapping the neural mechanisms behind psychedelic efficacy. Microdosing could also be explored as an adjunct to long-term psychotherapy predicated on the longitudinal cultivation of resilience and insight, a new paradigm that could compliment the acutely transformative model underlying high-dose psychedelic psychotherapy (Rosenbaum et al., 2018).

\section{Conclusion}

This study provides initial, correlational evidence for mental health and personality benefits associated with microdosing psychedelics. While anecdotal reports of microdosing benefits have existed for some time (Fadiman, 2011), this study marks the first formal study of the topic. Additionally, the use of a pre-registered study design sets a precedent for responsible and replicable psychedelic microdosing research. To add depth to the current discussion, a full epidemiological report (Rosenbaum et al., 2018) and a Grounded Theory analysis of qualitative outcomes (Anderson et al., 2018) are forthcoming. 
The results of the present study suggest that there is a significant relationship between microdosing experience and measures of mental health and flourishing including lower dysfunctional attitudes and negative emotionality, higher wisdom and open-mindedness, and higher creativity and affect-valence. These findings are the initial evidence that warrants RCTs to directly test safety and therapeutic efficacy. With approximately 30,000 users subscribing to the /r/microdosing subreddit and thousands more reading media reports on microdosing this growing community continues to explore microdosing and its effects. It is our hope that scientific reporting can help to clarify and inform the public about the nature of microdosing's putative effects and that this new paradigm helps shape future psychedelic research. We hope that researchers will draw on our shared resources (https://osf.io/g5cwy/) and pre-register studies of their own so that psychedelic science will be built upon strong research practices. Insights from these and other studies will form the backbone of future research into microdosing psychedelics.

\section{References}

Adler AD, Strunk DR and Fazio RH (2015) What Changes in Cognitive Therapy for Depression? An Examination of Cognitive Therapy Skills and Maladaptive Beliefs. Behavior Therapy 46(1). Special Series: Advances in Evidence-Based Intervention and Assessment Practices for Youth with an Autism Spectrum Disorder: 96-109. DOI: 10.1016/j.beth.2014.09.001.

Amsterdam J van, Opperhuizen A and Brink W van den (2011) Harm potential of magic mushroom use: A review. Regulatory Toxicology and Pharmacology 59(3): 423-429. DOI: 10.1016/j.yrtph.2011.01.006.

Anderson T, Christopher A, Petranker R, et al. (2018) Benefits and Drawbacks of Microdosing Psychedelics. Manuscript in preparation. Available at: osf.io/g5cwy.

Bogenschutz MP, Forcehimes AA, Pommy JA, et al. (2015) Psilocybin-assisted treatment for alcohol dependence: a proof-of-concept study. Journal of Psychopharmacology (Oxford, England) 29(3): 289-299. DOI: 10.1177/0269881114565144.

Carhart-Harris RL, Roseman L, Bolstridge M, et al. (2017) Psilocybin for treatment-resistant depression: fMRI-measured brain mechanisms. Scientific Reports 7(1): 13187. DOI: 10.1038/s41598-017-13282-7. 
de Graaf LE, Roelofs J and Huibers MJH (2009) Measuring Dysfunctional Attitudes in the General Population: The Dysfunctional Attitude Scale (form A) Revised. Cognitive Therapy and Research 33(4): 345-355. DOI: 10.1007/s10608-009-9229-y.

de Wit H and Phillips TJ (2012) Do initial responses to drugs predict future use or abuse? Neuroscience and Biobehavioral Reviews 36(6): 1565-1576. DOI: 10.1016/j.neubiorev.2012.04.005.

Domínguez-Clavé E, Soler J, Elices M, et al. (2016) Ayahuasca: Pharmacology, neuroscience and therapeutic potential. Brain Research Bulletin 126(Pt 1): 89-101. DOI: 10.1016/j.brainresbull.2016.03.002.

Dos Santos RG, Osório FL, Crippa JAS, et al. (2016) Antidepressive, anxiolytic, and antiaddictive effects of ayahuasca, psilocybin and lysergic acid diethylamide (LSD): a systematic review of clinical trials published in the last 25 years. Therapeutic Advances in Psychopharmacology 6(3): 193-213. DOI: 10.1177/2045125316638008.

Fadiman J (2011) The Psychedelic Explorer's Guide. Rochester, VT: Park Street Press.

Fredrickson BL (2004) The broaden-and-build theory of positive emotions. Philos Trans R Soc Lond B Biol Sci 359(1449): 1367-78. DOI: 10.1098/rstb.2004.1512.

Glück J, König S, Naschenweng K, et al. (2013) How to measure wisdom: content, reliability, and validity of five measures. Frontiers in Psychology 4. DOI: 10.3389/fpsyg.2013.00405.

Griffiths RR, Johnson MW, Richards WA, et al. (2011) Psilocybin occasioned mystical-type experiences: immediate and persisting dose-related effects. Psychopharmacology 218(4): 649-665. DOI: 10.1007/s00213-011-2358-5.

Griffiths RR, Johnson MW, Carducci MA, et al. (2016) Psilocybin produces substantial and sustained decreases in depression and anxiety in patients with life-threatening cancer: A randomized double-blind trial. Journal of Psychopharmacology (Oxford, England) 30(12): 1181-1197. DOI: 10.1177/0269881116675513.

Gunnarsson M, Gustavsson P, Tengström A, et al. (2008) Personality traits and their associations with substance use among adolescents. Personality and Individual Differences 45: 356360. DOI: 10.1016/j.paid.2008.05.004.

Halpern JH and Pope HG (1999) Do hallucinogens cause residual neuropsychological toxicity? Drug and Alcohol Dependence 53(3): 247-256. DOI: 10.1016/S0376-8716(98)00129-X.

Hardaway R, Schweitzer J and Suzuki J (2016) Hallucinogen Use Disorders. Child and Adolescent Psychiatric Clinics of North America 25(3): 489-496. DOI: 10.1016/j.chc.2016.03.006. 
Jarrett RB, Minhajuddin A, Borman PD, et al. (2012) Cognitive Reactivity, Dysfunctional Attitudes, and Depressive Relapse and Recurrence in Cognitive Therapy Responders. Behaviour Research and Therapy 50(5): 280-286. DOI: 10.1016/j.brat.2012.01.008.

Johnson MW, Garcia-Romeu A, Cosimano MP, et al. (2014) Pilot Study of the 5-HT2AR Agonist Psilocybin in the Treatment of Tobacco Addiction. Journal of psychopharmacology (Oxford, England) 28(11): 983-992. DOI: $10.1177 / 0269881114548296$.

Kometer M, Pokorny T, Seifritz E, et al. (2015) Psilocybin-induced spiritual experiences and insightfulness are associated with synchronization of neuronal oscillations. Psychopharmacology 232(19): 3663-3676. DOI: 10.1007/s00213-015-4026-7.

Krebs TS and Johansen P- $\varnothing$ (2013) Psychedelics and Mental Health: A Population Study. PLoS ONE 8(8). DOI: 10.1371/journal.pone.0063972.

Lahey BB (2009) Public Health Significance of Neuroticism. The American psychologist 64(4): 241-256. DOI: 10.1037/a0015309.

Leonard A (2015) How LSD Microdosing Became the Hot New Business Trip. In: Rolling Stone. Available at: https://www.rollingstone.com/culture/culture-news/how-lsdmicrodosing-became-the-hot-new-business-trip-64961/ (accessed 9 July 2018).

MacLean KA, Johnson MW and Griffiths RR (2011) Mystical Experiences Occasioned by the Hallucinogen Psilocybin Lead to Increases in the Personality Domain of Openness. Journal of psychopharmacology (Oxford, England) 25(11): 1453-1461. DOI: 10.1177/0269881111420188.

Matejka J, Glueck M, Grossman T, et al. (2016) The Effect of Visual Appearance on the Performance of Continuous Sliders and Visual Analogue Scales. In: Proceedings of the 2016 CHI Conference on Human Factors in Computing Systems, New York, NY, USA, 2016, pp. 5421-5432. CHI '16. ACM. DOI: 10.1145/2858036.2858063.

Moreno FA, Wiegand CB, Taitano EK, et al. (2006) Safety, tolerability, and efficacy of psilocybin in 9 patients with obsessive-compulsive disorder. The Journal of Clinical Psychiatry 67(11): 1735-1740.

Osório F de L, Sanches RF, Macedo LR, et al. (2015) Antidepressant effects of a single dose of ayahuasca in patients with recurrent depression: a preliminary report. Revista Brasileira De Psiquiatria (Sao Paulo, Brazil: 1999) 37(1): 13-20. DOI: 10.1590/1516-4446-20141496.

Rosenbaum D, Weissman C, Hapke E, et al. (2018) Microdosing psychedelic substances: demographics, psychiatric comorbidities, and comorbid substance use. Manuscript in preparation. Manuscript submitted for publication. Available at: osf.io/g5cwy.

Ross S, Bossis A, Guss J, et al. (2016) Rapid and sustained symptom reduction following psilocybin treatment for anxiety and depression in patients with life-threatening cancer: a 
randomized controlled trial. Journal of Psychopharmacology (Oxford, England) 30(12): 1165-1180. DOI: 10.1177/0269881116675512.

Silvia PJ (2011) Subjective scoring of divergent thinking: Examining the reliability of unusual uses, instances, and consequences tasks. Thinking Skills and Creativity 6(1): 24-30. DOI: 10.1016/j.tsc.2010.06.001.

Silvia PJ, Winterstein BP, Willse JT, et al. (2008) Assessing creativity with divergent thinking tasks: Exploring the reliability and validity of new subjective scoring methods. Psychology of Aesthetics, Creativity, and the Arts 2(2): 68-85. DOI: 10.1037/19313896.2.2.68.

Solon O (2016) Under pressure, Silicon Valley workers turn to LSD microdosing. Wired UK, 24 August. Available at: http://www.wired.co.uk/article/lsd-microdosing-drugs-siliconvalley (accessed 9 July 2018).

Soto CJ and John OP (2016) The Next Big Five Inventory (BFI-2): Developing and Assessing a Hierarchical Model With 15 Facets to Enhance Bandwidth, Fidelity, and Predictive Power. Article in Press. Available at: https://www.scopus.com/inward/record.uri?eid=2s2.0-84962720315\&partnerID=40\&md5=a060a5135d4ee7cfc25cb84c5118a272.

Strassman RJ (2016) DMT: The Spirit Molecule: A Doctor's Revolutionary Research into the Biology of Near-Death and Mystical Experiences. Rochester, VT: Inner Traditions.

Terracciano A, Löckenhoff CE, Crum RM, et al. (2008) Five-Factor Model personality profiles of drug users. BMC Psychiatry 8: 22. DOI: 10.1186/1471-244X-8-22.

Trull TJ and Sher KJ (1994) Relationship between the five-factor model of personality and Axis I disorders in a nonclinical sample. Journal of Abnormal Psychology 103(2): 350-360. DOI: $10.1037 / 0021-843 X .103 .2 .350$.

UNESCO Institute for Statistics (2011) International Standard Classification of Education (ISCED) 2011. Available at: http://uis.unesco.org/sites/default/files/documents/international-standard-classificationof-education-isced-2011-en.pdf.

Waldman A (2017) A Really Good Day: How Microdosing Made a Mega Difference in My Mood, My Marriage, and My Life. 1 edition. New York: Knopf. 